\title{
Avaliação de híbridos intervarietais de milho por meio de cruzamento dialélico parcial, considerando quatro ambientes
}

\section{Evaluation of intervarietal maize hybrids through partial diallel cross, considering four environments}

\author{
Deoclécio Domingos Garbuglio ${ }^{1 *}$; Pedro Mário de Araújo²
}

\begin{abstract}
Resumo
Foram avaliados 12 híbridos intervarietais de milho (Zea mays L.), cruzados em esquema dialélico parcial $3 \times 4$ mais as sete populações parentais e 3 testemunhas. Os ensaios foram implantados nas estações experimentais do Iapar em Londrina, Pato Branco, Ponta Grossa e Guarapuava. O objetivo foi avaliar um conjunto de sete populações no sentido de se estimar parâmetros que venham a auxiliar na escolha de materiais para posteriores trabalhos com seleção recorrente, extração de linhagens e síntese de compostos. Para a fonte Heterose as diferenças foram constatadas $(\mathrm{P}<0,01)$ para as variáveis produtividade de grãos e florescimento feminino, indicando que os cruzamentos foram superiores aos pais. Em relação aos valores de Capacidade Geral de Combinação para rendimento, os parentais do grupo-1, PC 9407 e BR 106, obtiveram como valores 36,7 e $171,1 \mathrm{~kg} \cdot \mathrm{ha}^{-1}$, respectivamente, e os parentais do grupos-2, PC 9701 e PC 9502, apresentaram valores de 131,1 e 56,8 kg.ha-1, respectivamente,. Para a Capacidade Específica de Combinação, os maiores valores foram para PC 9701 x BR 106 (205,4 kg.ha-1) e PC 9502 x PC 9407 (135,3 $\left.\mathrm{kg} \cdot \mathrm{ha}^{-1}\right)$, assim como as maiores médias de produtividade de grãos dos cruzamentos avaliados $(9.297 \mathrm{e}$ 9.018 kg.ha-1 ${ }^{-1}$, respectivamente). As populações PC 9701, BR 106, PC 9502 e PC 9407 são promissoras fontes para a extração de linhagens visando desenvolvimento de híbridos, síntese de compostos e trabalhos com seleção recorrente.
\end{abstract}

Palavras Chave: Zea mays L. Capacidade de combinação. Variedade de polinização aberta.

\begin{abstract}
In the summer season of 2004/2005, 12 intervarietal maize hybrids, crossed by the partial diallel scheme $3 \times 4$, the seven parental populations and three checks were evaluated at Iapar Experimental Stations in Londrina, Pato Branco, Ponta Grossa e Guarapuava. The objective was to identify crosses for future work with recurrent selection, inbred lines extraction and composite synthesis. For Heterosis variation cause, the differences were determined $(\mathrm{P}<0.01)$ for traits grain yield and female flowering, indicating that crosses were superior in relation to parentals. Regarding General Combining Ability values for yield, the parentals from Group-1, PC 9407 and BR 106, showed values of $36.7 \mathrm{~kg} \cdot \mathrm{ha}^{-1}$ and $171.1 \mathrm{~kg} \cdot \mathrm{ha}^{-1}$, respectively, and the parentals from Group-2 presented values of $131.1 \mathrm{~kg} . \mathrm{ha}^{-1}$ and $56.8 \mathrm{~kg} \cdot \mathrm{ha}^{-1}$, respectively,. For Specific Combining Ability, the largest values were from PC 9701 x BR $106\left(205.4 \mathrm{~kg} \cdot \mathrm{ha}^{-1}\right)$ e PC 9502 x PC 9407 (135.3 kg.ha-1) and, the largest values of grain yield average (9297 e $9018 \mathrm{~kg} \cdot \mathrm{ha}^{-1}$, respectively). The populations PC 9701, BR 106, PC 9502 and PC 9407 are promising sources for inbred lines extraction aiming to superior maize hybrids development, composite synthesis and recurrent selection.

Key words: Zea mays $L$.. Combining ability. Open pollinated variety.
\end{abstract}

\footnotetext{
${ }^{1}$ Eng $^{\circ}$ Agr ${ }^{\circ}$, Pós-Graduando em Genética e Melhoramento de Plantas - ESALQ / USP . Colaborador, IAPAR, Área de Melhoramento e Genética Vegetal - Programa Milho. deocleciodg@yahoo.com.br

2 IAPAR, Pesquisador Dr. Área de Melhoramento e Genética Vegetal - Programa Milho, pmaraujo@iapar.br

Autor para correspondência
} 


\section{Introdução}

O Paraná na safra de verão 2004/2005, segundo levantamento realizado pela Secretaria Agricultura e Abastecimento do Paraná (PARANÁ, 2005), produziu cerca de 8,07 milhões de toneladas de milho em 1,27 milhões de hectares colhidos. A produtividade média de grãos, que foi recorde na safra 2002/2003, atingindo a marca de 5600 kg.ha ${ }^{-1}$, elevouse para $5972 \mathrm{~kg} . \mathrm{ha}^{-1}$ na safra atual, demonstrando o alto nível de tecnologia empregado pelos agricultores paranaenses.

Segundo Corrêa et al. (2004), a região sul atingiu uma produção de $18.802,9$ mil toneladas na soma das $1^{\mathrm{a}}$ e $2^{\mathrm{a}}$ safras 2003/2004, sendo o Paraná o maior produtor com 11.104,4 mil toneladas. No entanto esta marca está $18,7 \%$ abaixo daquela atingida na safra anterior, 2002/2003, que foi de 13.657,2 mil toneladas.

O melhoramento de milho é dirigido essencialmente para atender duas alternativas: a)obtenção de variedade de polinização livre; b)obtenção de híbridos. A seleção recorrente recíproca busca, basicamente, aumentar a resposta heterótica entre duas populações.

É amplamente reconhecido que a obtenção de híbridos superiores de milho depende em grande parte das populações parentais das quais serão extraídas as linhagens que vão compor esse híbrido (ARAÚJO; PATERNIANI, 1999). Segundo Russel, Blackburn e Lamkey (1992), em um programa voltado para esse fim, o desenvolvimento dessas linhagens que apresentem desempenho superior em combinações híbridas é o principal objetivo do melhorista de milho. Portanto, em um programa de desenvolvimento de híbridos, a escolha do germoplasma a ser utilizado como fonte para obtenção de linhagens é de fundamental importância visando capitalizar os efeitos de heterose no híbrido (GERAGE; ARAÚJO, 2002). No entanto, como citado por Vencovsky (1987), a probabilidade de se obter uma linhagem agronômicamente boa, vai depender da frequiência dos alelos favoráveis dessa população. A elevação dessas freqüências se dá por meio do melhoramento dessas populações via seleção recorrente intra ou interpopulacional. Como o número de populações dos programas é muito grande, se faz necessário uma seleção prévia daquelas que resultem em melhores combinações híbridas para, posteriormente, serem submetidas aos diferentes processos de seleção.

A avaliação de cruzamentos intervarietais possibilita a escolha de populações para trabalhos posteriores com seleção recorrente intra ou interpopulacional (seleção recorrente recíproca), formação de compostos ou utilização comercial do $\mathrm{F}_{1}$ (HALLAUER; MIRANDA FILHO, 1988; CROSSA; TABA; WELHAUSEN, 1990). Comstock, Robinson e Harvey (1949) sugeriram a seleção recorrente recíproca como alternativa para incrementar a resposta heterótica entre duas populações de milho específicas. O método explora conjuntamente os efeitos de capacidade geral e específica de combinação e visa a obtenção de combinações híbridas superiores através do desenvolvimento de linhagens divergentes entre as duas populações.A análise de cruzamentos dialélicos entre variedades (populações) permite a predição de novos compostos. Estes são originários do intercruzamento entre um grupo de populações já conhecido visando a formação de uma nova população.

O termo dialelo vem sendo empregado para expressar um conjunto de $\mathrm{p}(\mathrm{p}-1) / 2$ híbridos, resultante do cruzamento entre p parentais (linhagens, variedades, clones), podendo incluir, além dos respectivos parentais, os híbridos recíprocos e/ou outras gerações relacionadas tais como $\mathrm{F}_{2} \mathrm{e}$ retrocruzamentos, entre outros. Segundo Cruz e Regazzi (1994), as metodologias de análise dialélica têm por finalidade analisar o delineamento genético, provendo estimativas de parâmetros úteis na seleção de parentais para hibridação e no entendimento dos efeitos genéticos envolvidos na determinação dos caracteres.

Entre as metodologias de análise dialética mais comumente utilizadas citam-se a proposta por Griffing 
(1956), na qual são estimados os efeitos de capacidade geral e específica de combinação (CGC e CEC, respectivamente). Na metodologia proposta por Gardner e Eberhart (1966), são estimados os efeitos de variedades $\left(\mathrm{v}_{\mathrm{i}}\right.$ e $\mathrm{v}_{\mathrm{j}}$ ) e de heterose varietal $\left(h_{i}\right.$ e $\left.h_{j}\right)$; e a proposta por Hayman (1954), que dá informações sobre o mecanismo básico de herança do caráter em estudo, dos valores genéticos dos parentais utilizados e do limite de seleção. Adaptações do modelo de Griffing (1956) e de Gardner e Eberhart (1966), para dialelos parciais, têm possibilitado maximizar as informações sobre os grupos estudados com um número menor de cruzamentos do que os requeridos no dialelo completo. Para análises onde são considerados os efeitos de diferentes ambientes, destacam-se as adaptações propostas por Morais et al. (1991), quanto ao modelo II de Gardner e Eberhart (1966), para dialelos completos, e a proposta por Oliveira et al. (1987) para análises de dialelos parciais entre populações, para análises combinadas em ambientes distintos.
O presente estudo teve por objetivo avaliar um conjunto de sete populações em quatro ambientes, cruzadas em esquema dialélico parcial ( $3 \times 4)$, no sentido de se estimar parâmetros que venham a auxiliar na escolha de materiais para posteriores trabalhos com seleção recorrente, extração de linhagens e síntese de compostos.

\section{Material e Métodos}

Durante a safra de verão 2004/2005 foram avaliados 12 híbridos intervarietais, resultado do cruzamento entre sete populações pertencentes ao programa de melhoramento genético de milho do IAPAR, em esquema dialélico parcial $3 \times 4$, sem recíprocos, mais as sete populações parentais e três testemunhas, totalizando 22 tratamentos. Os materiais pertencentes a cada grupo de avaliação são apresentados na Tabela 1.

Tabela 1. Disposição das sete populações nos grupos submetidos ao intercruzamento para a formação de um Dialelo Parcial (3 x 4), e referidas características. Safra verão 2004/2005

\begin{tabular}{lcl}
\hline Grupos de Cruzamento & Genótipos & \multicolumn{1}{c}{ Características } \\
\hline Grupo I & PC 9407 & Ciclo precoce, porte baixo, grãos dentados. Origem: IAPAR \\
& PC 9501 & Ciclo precoce, porte baixo, grãos dentados. Origem: IAPAR \\
BR 106 & Ciclo normal, porte alto, grãos dentados. Origem: Embrapa Milho e Sorgo \\
& & \\
Grupo II & PC 9701 & Ciclo precoce, porte baixo, grãos semi-dentados. Origem: IAPAR \\
& PC 9502 & Ciclo precoce, porte baixo, grãos semi-dentados. Origem: IAPAR \\
& PC 9702 & Ciclo precoce, porte baixo, grãos semi-dentados. Origem: IAPAR \\
PC 9703 & Ciclo precoce, porte baixo, grãos semi-dentados. Origem: IAPAR \\
\hline
\end{tabular}

O delineamento utilizado foi o de blocos ao acaso com duas repetições. A parcela útil foi constituída de 2 linhas de 5 metros de comprimento com $0,80 \mathrm{~m}$ de espaçamento entre linhas, contendo 5 plantas por metro linear após o desbaste. As testemunhas foram os híbridos IPR 115 - híbrido simples sintetizado no programa de melhoramento genético do Iapar; $\mathrm{P}$ 30F33 - híbrido simples da empresa Pionner e BRS 1010 - híbrido simples da Embrapa. Os experimentos foram implantados nas estações do Iapar localizadas em Londrina, Ponta Grossa, Pato Branco e
Guarapuava. As análises estatísticas foram realizadas com auxílio dos programas GENES (CRUZ, 1997) e MAPGEN (FERREIRA, 1993).

Foram avaliados os seguintes caracteres:

AP: Altura de Planta - medida da superfície do solo à curvatura da folha bandeira, em cm; AE: Altura de espiga - medida da superfície do solo até o ponto de inserção da espiga superior, em cm; FLOR: Florescimento Feminino - medida em número de dias necessários para a liberação dos estigmas em mais 
de 50\% das plantas da parcela; PROL: Prolificidade - expressa pelo número de espigas em relação ao número de plantas do estande final; REND: produtividade de grãos corrigido para kg.ha-1, padronizado a $14,5 \%$ de umidade.

As análises de variância individuais foram realizadas seguindo-se o modelo de blocos ao acaso com testemunhas adicionais $\left(\mathrm{Y}_{\mathrm{ij}}=\mu+\mathrm{T}_{\mathrm{i}}+\mathrm{B}_{\mathrm{j}}+\varepsilon_{\mathrm{ij}}\right)$ proposto por Cruz e Carneiro (2003), onde $\mathrm{Y}_{\mathrm{ij}}$ : observação do i-ésimo tratamento (família ou testemunha) no j-ésimo bloco; 1/4: média geral por local; $\mathrm{T}_{\mathrm{i}}$ : efeito do i-ésimo tratamento. Para i-1,2...g, tem-se o efeito aleatório das familias e para $\mathrm{i}=\mathrm{g}+1$, $\mathrm{g}+2, \ldots \mathrm{g}+\mathrm{t}$ tem-se o efeito fixo de testemunhas; $\mathrm{B}_{\mathrm{j}}$ : efeito do bloco $\mathrm{j} ; \mu_{\mathrm{ij}}$ : erro aleatório.

As análises estatísticas conjuntas dos dados seguiram o modelo fatorial simples com testemunhas adicionais, tendo como fixos os genótipos e, como aleatórios, os ambientes (CRUZ, 1997).

Após análise estatística dos dados, os mesmos foram submetidos à análise dialélica conjunta, seguindo o modelo proposto por Gardner e Eberhart (1966), com adaptação de Miranda Filho e Geraldi (1984) aplicável a dialelos parciais completos e a readaptação proposta por Oliveira et al. (1987), onde são considerados vários ambientes (Tabela 2).

Tabela 2. Esquema da análise de variância conjunta de cruzamentos dialélicos parciais, envolvendo J mais J' populações e seus JJ' híbridos, em I ambientes (OLIVEIRA et al., 1987).

\begin{tabular}{|c|c|c|}
\hline FV & G.L. & S.Q. \\
\hline Ambientes (A) & (I-1) & $\mathrm{SQ}_{\mathrm{A}}$ \\
\hline Populações (P) & $\left(\mathrm{J}+\mathrm{J}^{\prime}+\mathrm{JJ}^{\prime}-1\right)$ & $\mathrm{SQ}_{\mathrm{P}}$ \\
\hline Parentais $1(\mathrm{P} 1)$ & $(\mathrm{J}-1)$ & $\mathrm{SQ}_{\mathrm{P} 1}$ \\
\hline Parentais $2(\mathrm{P} 2)$ & $\left(J^{\prime}-1\right)$ & $\mathrm{SQ}_{\mathrm{P} 2}$ \\
\hline Grupos (Gru) & 1 & $\mathrm{SQ}_{\mathrm{G}}$ \\
\hline Heterose $(\mathrm{H})$ & $\mathrm{JJ}{ }^{\prime}$ & - \\
\hline Het. média (HM) & 1 & $\mathrm{SQ}_{\mathrm{HM}}$ \\
\hline Het. Parentais 1 (HP1) & $\mathrm{J}-1$ & $\mathrm{SQ}_{\mathrm{HP} 1}$ \\
\hline Het. Parentais 2 (HP2) & $J^{\prime}-1$ & $\mathrm{SQ}_{\mathrm{HP2}}$ \\
\hline Het. específica (HE) & $(\mathrm{J}-1)(\mathrm{J} '-1)$ & $\mathrm{SQ}_{\mathrm{HE}}$ \\
\hline Interação A x P & $(\mathrm{I}-1)\left(\mathrm{J}+\mathrm{J}^{\prime}+\mathrm{JJ}^{\prime}-1\right)$ & $\mathrm{SQ}_{\mathrm{AP}}$ \\
\hline A x P1 & $(\mathrm{I}-1)(\mathrm{J}-1)$ & $\mathrm{SQ}_{\mathrm{AP} 1}$ \\
\hline A $x$ P2 & $(\mathrm{I}-1)(\mathrm{J} '-1)$ & $\mathrm{SQ}_{\mathrm{AP2}}$ \\
\hline$A \times G$ & (I-1) & $\mathrm{SQ}_{\mathrm{AG}}$ \\
\hline $\mathrm{A} \times \mathrm{H}$ & $(\mathrm{I}-1) \mathrm{JJ}$ & - \\
\hline A x HM & (I-1) & $\mathrm{SQ}_{\mathrm{AHM}}$ \\
\hline A x HP1 & $(\mathrm{I}-1)(\mathrm{J}-1)$ & $\mathrm{SQ}_{\mathrm{AHP1}}$ \\
\hline A $x$ HP2 & (I-1) (J'-1) & $\mathrm{SQ}_{\mathrm{AHP2}}$ \\
\hline $\mathrm{A} \times \mathrm{HE}$ & $(\mathrm{I}-1)(\mathrm{J}-1)(\mathrm{J} '-1)$ & $\mathrm{SQ}_{\mathrm{AHE}}$ \\
\hline $\begin{array}{l}\text { Resíduo combinado } \\
\text { gi= número de graus de libe }\end{array}$ & $\begin{array}{c}\text { Sigi } \\
\text { mo ambiente }\end{array}$ & \\
\hline
\end{tabular}

O modelo para cruzamentos dialélicos, proposto por Miranda Filho e Geraldi (1984), inclui dois grupos de populações e os híbridos resultantes dos cruzamentos intervarietais de grupos distintos. Quando os ensaios são repetidos em vários ambientes, o modelo adaptado passa a ser: onde:

$$
\begin{aligned}
& \mathrm{Y}_{\mathrm{ijj}}=\mu+\alpha \mathrm{d}+\ell_{\mathrm{i}}+\frac{1}{2}\left(\mathrm{v}_{\mathrm{j}}+\mathrm{v}_{\mathrm{j}^{\prime}}\right)+ \\
& \theta\left(\overline{\mathrm{h}}+\mathrm{h}_{\mathrm{j}}+\mathrm{h}_{\mathrm{j}^{\prime}}+\mathrm{s}_{\mathrm{jj} j^{\prime}}\right)+\alpha \ell \mathrm{d}_{\mathrm{i}}+\frac{1}{2}\left(\ell \mathrm{v}_{\mathrm{ij}}+\ell \mathrm{v}_{\mathrm{ij}}\right)+ \\
& \theta\left(\ell \overline{\mathrm{h}}+\ell \mathrm{h}_{\mathrm{j}}+\ell \mathrm{h}_{\mathrm{j}^{\prime}}+\ell \mathrm{s}_{\mathrm{jj}}\right)+\overline{\mathrm{e}}_{\mathrm{ijj}}
\end{aligned}
$$


$\mathrm{Y}_{\mathrm{ijj}}$ : Média do híbrido resultante do cruzamento entre a j-ésima população do grupo 1 e a j’ população do grupo 2 no i-ésimo ambiente, sendo $q=1$ e a=0 para híbridos. Para representar os pais (populações base) no i-ésimo ambiente, $\mathrm{Y}_{\mathrm{ijj}}$, é substituido por $\mathrm{Y}_{\mathrm{ijj}}$ ou $Y_{i j ' j}$, para populações dos grupos 1 ou 2, respectivamente. Para $Y_{\mathrm{ijj}}, \alpha=+1$ e $\theta=0$; e para $\mathrm{Y}_{\mathrm{ij} j \mathrm{j}}$, $\alpha=-1$ e $q=\theta ; \bar{\mu}$ : Média das médias dos dois grupos de populações para todos os ambientes; d: Medida da diferença entre as médias dos dois grupos de variedades; $\ell_{\mathrm{i}}$ Efeito do i-ésimo ambiente; $v_{\mathrm{j}}$ e $\mathrm{v}_{\mathrm{j}}$ : São os efeitos das populações pertencentes aos grupos 1 e 2 , respectivamente; $\overline{\mathrm{h}}$ Heterose média de todos os cruzamentos; $h_{i}$ e $h_{j}$ : Efeitos das heteroses de variedades para os grupos 1 e 2 , respectivamente; $\mathrm{s}_{\mathrm{j} j}$ : Heterose específica do jj' -ésimo híbrido; $\overline{\mathrm{e}}_{\mathrm{ijj}}$ : Erro experimental associado a $\mathrm{Y}_{\mathrm{ijj}}$,

\section{Resultados e Discussão}

Através das análises individuais de variância (Tabela 3), para a localidade de Londrina, foi detectada alta significância $(\mathrm{P}<0,01)$ pelo teste $\mathrm{F}$ para a Fonte de variação (FV) tratamentos (Trat) com relação as variáveis FLOR, AP, PROL, REND. Para as FV Genótipos (G) e Testemunhas (test) com as variáveis FLOR e PROL; e para a FV G vs test para as variáveis FLOR, AP, REND. Não foi detectada significância $(\mathrm{P}>0,05)$ para a $\mathrm{FV}$ Test e as variáveis AE e REND, assim como para G vs Test junto à variável AE. Com relação as demais observações foi detectada significância ao nível de 5\%.

Para a localidade de Guarapuava foi verificada significância para as FV Trat e G com relação a variável FLOR e para as FV Trat, G, Test e G vs Test para a variável REND. Na análise individual para a localidade de Ponta Grossa, foi detectada significância $(\mathrm{P}<0,01)$ para a FV Trat com relação as variáveis FLOR e REND; para a FV G em relação a variável FLOR e para a FV G vs Test com relação as variáveis PROL e REND. Significâncias ao nível de $5 \%$ foram detectadas para a FV G e a variável REND e para a FV Test e a variável FLOR (Tabela 3).

Para a localidade de Pato Branco observou-se significância pelo teste $\mathrm{F}(\mathrm{P}<0,01)$ para todas as $\mathrm{FV}$ para a variável REND. Para as FV Trat e G foi detectada significância ao nível de 5\% para a variável AP e para Test com relação a variável AE. Para as demais FV e variáveis não foi detectada significância ( $\mathrm{P}>0,05)$ (Tabela 3).

Nas análises individuais pôde ser constatado que os CV\% foram considerados baixos, implicando em uma alta precisão experimental. As médias para REND das testemunhas se apresentaram superiores as médias gerais e dos genótipos (todos os tratamentos excluindo-se as testemunhas), na média dos quatro ambientes de avaliação (Tabela 4). Para PROL e AE não houve grandes variações, estando as médias ao redor de uma espiga por planta e $127 \mathrm{~cm}$, respectivamente (Tabela 4 ). As diferenças médias quanto a variável FLOR podem estar associadas, mais diretamente, às diferenças de temperatura média durante o período para as localidades em questão, sendo que em Londrina observa-se maiores temperaturas em relação à Guarapuava, Ponta Grossa e Pato Branco, implicando em uma redução no ciclo de florescimento (Tabela 3).

Foi realizada a análise conjunta através do modelo fatorial simples com testemunhas adicionais (Tabela 4), tendo como fixos os genótipos e como aleatórios os ambientes (CRUZ, 1997). 
Tabela 3. Quadrados médios obtidos pelas análises de variância em blocos ao acaso com testemunhas adicionais, para as variáveis FLOR, AP, AE, PROL, REND. Londrina, Guarapuava, Ponta Grossa e Pato Branco. 2004/2005

\begin{tabular}{|c|c|c|c|c|c|c|}
\hline \multicolumn{7}{|c|}{ LONDRINA } \\
\hline \multirow[t]{2}{*}{ FV } & \multirow{2}{*}{ GL } & FLOR & $\mathbf{A P}$ & $\mathbf{A E}$ & PROL & ${ }^{1}$ REND \\
\hline & & (dias) & $(\mathrm{cm})$ & $(\mathrm{cm})$ & (esp/pl) & $\left({ }^{2} \mathrm{~kg} \cdot \mathrm{ha}^{-1}\right)$ \\
\hline TRATAMENTOS & 21 & $2,23 * *$ & $198,23 * *$ & $134,39 *$ & $0,035^{* *}$ & $104,94 * *$ \\
\hline Genótipos & 18 & $1,82 * *$ & $158,24 *$ & $144,64^{*}$ & $0,016^{* *}$ & $97,62 *$ \\
\hline Testemunha & 2 & $4,17 * *$ & $329,17^{*}$ & 29,17 & $0,215^{* *}$ & 26,80 \\
\hline G vs Test & 1 & $5,55^{* *}$ & $656,34 * *$ & 160,27 & $0,025^{*}$ & $392,98 * *$ \\
\hline RESÍDUO & 21 & 0,60 & 62,51 & 50,22 & 0,004 & 35,40 \\
\hline Média & - & 65 & 251 & 133 & 1.00 & 8766 \\
\hline CV\% & - & 1,19 & 3,15 & 5,32 & 6,59 & 6,79 \\
\hline \multicolumn{7}{|c|}{ GUARAPUAVA } \\
\hline TRATAMENTOS & 21 & $16,69 * *$ & 78,09 & 128,98 & 0,004 & $177,46 * *$ \\
\hline Genótipos & 18 & $18,12 * *$ & 74,38 & 130,74 & 0,004 & $112,69 * *$ \\
\hline Testemunha & 2 & 6,17 & 116,67 & 66,67 & 0,002 & $367,47 * *$ \\
\hline G vs Test. & 1 & 11,93 & 67,68 & 221,90 & 0,001 & $963,49 * *$ \\
\hline RESÍDUO & 21 & 4,26 & 77,27 & 91,14 & 0,005 & 16,72 \\
\hline Média & - & 76 & 189 & 137 & 1.02 & 7954 \\
\hline CV\% & - & 2,73 & 4,65 & 6,95 & 6,78 & 5,14 \\
\hline
\end{tabular}

\begin{tabular}{|c|c|c|c|c|c|c|c|}
\hline \multicolumn{8}{|c|}{ PONTA GROSSA } \\
\hline IRATAMENTOS & 21 & 16,88 & $* *$ & 79,66 & 117,88 & 0,0015 & $131,85 * *$ \\
\hline Genótipos & 18 & 18,55 & $* *$ & 85,46 & 135,73 & 0,0006 & $89,52 *$ \\
\hline Testemunha & 2 & 9,50 & $*$ & 66,67 & 4,17 & 0,0002 & 57,12 \\
\hline G vs Test. & 1 & 1,58 & & 1,39 & 23,93 & $0,0196 * *$ & $1043,12 * *$ \\
\hline RESÍDUO & 21 & 2,30 & & 63,12 & 115,89 & 0,0010 & 32,34 \\
\hline Média & - & 75 & & 225 & 119 & 0.96 & 7962 \\
\hline $\mathrm{zV} \%$ & - & 2,02 & & 3,54 & 9,08 & 3,24 & 7,14 \\
\hline
\end{tabular}

\begin{tabular}{lcccccc}
\hline \multicolumn{7}{c}{ PATO BRANCO } \\
\hline TRATAMENTOS & 21 & 3,29 & $358,90^{*}$ & 243,73 & 0,003 & $215,14^{* *}$ \\
Genótipos & 18 & 3,44 & $359,58^{*}$ & 214,67 & 0,002 & $136,80^{* *}$ \\
Testemunha & 2 & 2,67 & 387,50 & $487,50^{*}$ & 0,001 & $462,18^{* *}$ \\
G vs Test. & 1 & 1,95 & 289,44 & 279,33 & 0,020 & $1131,13^{* *}$ \\
RESÍDUO & 21 & 2,66 & 171,52 & 126,65 & 0,005 & 40,36 \\
Média & - & 71 & 217 & 119 & 1.03 & 9694 \\
CV\% & - & 2,31 & 6,05 & 9,47 & 6,79 & 6,55 \\
\hline
\end{tabular}

1; QM para a variável REND x $10^{5}$

2; REND corrigido para kg.ha- ${ }^{-1}$ a 14,5\% de umidade

**; significativo a $1 \%, *$; significativo a $5 \%$ pelo teste $\mathrm{F}$ 
Tabela 4. Quadrados médios obtidos pela análise de variância conjunta dos dados, seguindo o modelo fatorial simples com testemunhas adicionais, para as localidades de Londrina, Guarapuava, Ponta Grossa e Pato Branco. 2004/2005

\begin{tabular}{lcccccc}
\hline \multicolumn{1}{c}{ FV } & GL & ${ }^{1}$ REND & FLOR & AP & AE & PROL \\
\hline Tratamentos (Trat) & 21 & $49,73 * *$ & $26,89 * *$ & $406,84 * *$ & $385,77 * *$ & 0,013 \\
Genótipos (G) & 18 & $31,59 * *$ & $29,25 * *$ & $394,91 * *$ & $138,54 * *$ & 0,007 \\
Testem. (Test) & 2 & $67,35 *$ & $18,50 *$ & 379,17 & 604,21 & 0,046 \\
Grupos (Gru) & 1 & $341,02 * *$ & 1,16 & 676,96 & $4144,02 *$ & $0,056 *$ \\
Ambientes (Amb) & 3 & $300,56 * *$ & $991,11 * *$ & $29073,49 * *$ & $84,12 * *$ & $0,043 *$ \\
Trat x Amb & 63 & 4,40 & $4,49 * *$ & 102,68 & 80,01 & $0,010 * *$ \\
G x Amb & 54 & 4,02 & $4,53 * *$ & 94,25 & 149,65 & 0,005 \\
Test x Amb & 6 & $8,00 *$ & 2,44 & 173,61 & 27,07 & $0,058 * *$ \\
Gru x Amb & 3 & 4,02 & $7,88 * *$ & 112,63 & 95,98 & 0,003 \\
Resíduo & 84 & 3,12 & 1,79 & 93,60 & 95,98 & 0,004 \\
& & & & & & \\
MÉDIA geral & - & 8594 & 72 & 220 & 127 & 1,00 \\
MÉDIA genótipos & - & 8419 & 72 & 221 & 128 & 0,99 \\
MÉDIA testemunhas & - & 9702 & 72 & 215 & 122 & 1,04 \\
CV(\%) & - & 6,50 & 1,87 & 4,39 & 7,72 & 6,11 \\
\hline
\end{tabular}

${ }^{1}$ QM para a variável REND x $10^{5}$

${ }^{2}$ corrigido para kg.ha-1 a $14,5 \%$ de umidade

** significativo a $1 \%, *$; significativo a $5 \%$ pelo teste $\mathrm{F}$.

Observou-se para as FV Trat, G e Ambientes (Amb) diferenças significativas pelo teste $\mathrm{F}(\mathrm{P}<0,01)$ com relação as variáveis REND, FLOR, AP e AE, assim como para a FV Grupos (Gru) com a variável REND, Trat $x$ Amb com as variáveis FLOR e PROL, G x Amb e Gru x Amb com a variável FLOR e para a FV Test x Amb com variável PROL. Significâncias ao nível de $5 \%$ pelo teste $\mathrm{F}$ foram detectadas para a
FV Test com as variáveis REND e FLOR, Gru com as variáveis AE e PROL, Amb com a variável PROL e Test x Amb para a variável REND. Para as demais observações não foram detectadas quaisquer significâncias (P>0,05) (Tabela 4).

Após foi realizada a análise dialélica conjunta (Tabela 5), segundo a adaptação proposta por Oliveira et al. (1987) considerando vários ambientes. 
Tabela 5. Quadrados médios obtidos pela análise dialélica conjunta, aplicável a dialelo parciais completos em vários ambientes (OLIVEIRA et al.,1987), para as localidades de Londrina, Guarapuava, Ponta Grossa e Pato Branco. 2004/ 2005

\begin{tabular}{lcrrrrl}
\hline & GL.V. & \multicolumn{1}{c}{${ }^{1}$ REND } & FLOR & AP & AE & PROL \\
\cline { 3 - 8 } & 3 & $260,0 * *$ & $843,6 * *$ & $25925,6 * *$ & $3688,7 * *$ & $0,040 * *$ \\
\hline Ambientes (A) & 18 & $31,6 * *$ & $29,5 * *$ & $399,1 * *$ & $385,3 * *$ & 0,007 \\
Populações (P) & 2 & 7,1 & $145,0 * *$ & $1242,6 * *$ & $999,6 * *$ & $0,030 * *$ \\
Parentais 1 (P1) & 3 & $10,0 *$ & $20,5 * *$ & 193,5 & $465,4 * *$ & 0,000 \\
Parentais 2 (P2) & 1 & $39,3 * *$ & $75,9 * *$ & $2367,4 * *$ & $1676,6 * *$ & 0,011 \\
Grupos (Gru) & 12 & $40,4 * *$ & $8,7 * *$ & 145,8 & 155,3 & 0,004 \\
Heterose (H) & 1 & $389,7 * *$ & 2,2 & 175,1 & 39,4 & $0,018 *$ \\
Het. média (HM) & 2 & $38,4 * *$ & 6,4 & 126,6 & 82,6 & 0,001 \\
Het. Parentais 1 (HP1) & 3 & 2,8 & 0,8 & 129,9 & 123,5 & 0,001 \\
Het. Parentais 2 (HP2) & 6 & 1,7 & 14,4 & 155,3 & 214,7 & 0,004 \\
Het. específica (HE) & 54 & $4,0 *$ & $4,9 * *$ & 94,3 & 81,7 & 0,005 \\
Interação A x P & 6 & $8,7 * *$ & 14,9 & 28,3 & 115,8 & $0,017 * *$ \\
A x P1 & 9 & $5,6 *$ & 2,7 & 103,8 & 80,6 & 0,006 \\
A x P2 & 3 & 4,3 & $10,0 * *$ & 95,1 & 155,6 & 0,004 \\
A x G & 36 & 2,8 & 3,3 & 102,8 & 70,1 & 0,003 \\
A x H & 3 & 2,1 & $7,7 *$ & 87,8 & 225,5 & 0,002 \\
A x HM & 6 & $7,0 *$ & 3,2 & 143,1 & 48,9 & 0,004 \\
A x HP1 & 9 & 3,1 & 1,8 & 115,4 & 59,3 & 0,004 \\
A x HP2 & 18 & 1,5 & 3,4 & 85,6 & 56,7 & 0,003 \\
A x HE & 72 & 2,60 & 2,1 & 96,0 & 108,1 & 0,004 \\
Resíduo combinado & & & & & &
\end{tabular}

${ }^{1}$ QM para a variável REND x $10^{5}$

${ }^{2}$ corrigido para kg.ha- ${ }^{-1}$ a $14,5 \%$ de umidade

** significativo a $1 \%, *$; significativo a $5 \%$ pelo teste $\mathrm{F}$.

Para a FV Ambientes (A) foi detectada significância pelo teste $\mathrm{F}(\mathrm{P}<0,01)$ para todas as variáveis analisadas indicando, assim como na análise conjunta, haver diferenças entre os ambientes (Tabela 5). Para o conjunto de populações (P) avaliadas verificaram-se diferenças significativas para as variáveis REND, FLOR, AP e AE. Dentro do grupo de parentais 1 (P1), observou-se que estes apresentaram comportamento diferenciado parar todos os caracteres avaliados, exceto REND. Já para o grupo de parentais 2 (P2) foi detectada significância para as variáveis REND, FLOR e AE, enquanto que para as variáveis AP e PROL não foram detectadas diferenças significativas (Tabela 5).

Para a FV Grupos (Gru) detectou-se significância $(\mathrm{P}<0,01)$ para as variáveis REND, FLOR, AP e AE.
Para a FV Heterose Total $(\mathrm{H})$ e sua decomposição em Heterose Média (HM) e Heterose de variedades no grupo 1 (HV1) observou-se significância $(\mathrm{P}<0,01)$ para a variável REND, assim como em $\mathrm{H}$ para a variável FLOR. Ainda para HM, detectou-se significância ao nível de 5\% para PROL (Tabela 5). Araújo e Miranda Filho (2001) não encontraram diferenças significativas para heterose de variedades quanto às variáveis REND, FLOR, AP e AE e, para heterose específica, somente foi detectada significância para a variável AP. Para as demais variáveis, com relação a $\mathrm{H}$ e suas decomposições, não houve diferenças significativas que pudessem ser detectadas ao nível testado ( $\mathrm{P}>0,05)$ (Tabela 5). A não significância para as FV HM e HE (Heterose específica), com relação as variáveis FLOR, AP e 
AE, indica que os grupos, quando cruzados não apresentam potencial heterótico para esses caracteres. Ainda, as significâncias verificadas para $\mathrm{P} 1$, com relação às variáveis $\mathrm{AP}$ e $\mathrm{AE}$, e $\mathrm{P} 2$, com relação à $\mathrm{AE}(\mathrm{P}<0,01)$, podem ser explicadas com base no fato de existir uma predominância de efeitos aditivos e dominância apenas parcial para essas variáveis (COMSTOCK; ROBINSON, 1948; GARDNER; HARVEY; COMSTOCK, 1953; GORGULHO; MIRANDA FILHO, 2001).

Os efeitos de dominância interpopulacional tiveram maior importância que os efeitos de dominância intrapopulacional, podendo ser constatado na decomposição da soma de quadrados de populações, ou também chamada de soma de quadrados de entradas, onde $85,3 \%$ deve-se a $\mathrm{H}$; 2,5\% para o efeito de P1; 5,3\% ao efeito de P2 e $6,9 \%$ ao efeito de Gru. Estes resultados diferem dos encontrados por Araújo e Miranda Filho (2001), onde os efeitos de dominância intrapopulacional apresentaram maior importância.

A respeito da $\mathrm{HM}$, o fato desta ser significativa para REND, é um indicativo de que as populações em cruzamento foram superiores quando comparadas às populações per se. Ainda com relação a partição de $\mathrm{H}$ verificou-se que $80,3 \%$ foi devido ao efeito de HM; 15,8\% à heterose de parentais 1 (HP1); 1,7\% à heterose de parentais $2(\mathrm{HP} 2)$ e 2,1\% para heterose específica (HE). Gama et al (1995) constataram diferenças significativas para todas as fontes de variação para os caracteres AP e AE divergindo, em parte, dos resultados aqui obtidos.

Para os efeitos de interação de ambiente com as demais FV, observou-se em A x P diferenças ao nível de $5 \%$ para REND e a $1 \%$ para FLOR. Para A x P1 detectou-se significância $(\mathrm{P}<0,01)$ para REND e PROL enquanto que para A x P2 a significância foi detectada a 5\% somente para REND. Com relação a interação A x G (Tabela 5), houve diferenças significativas ao nível de $1 \%$ para FLOR e para A x HM essa diferença foi verificada ao nível de $5 \%$. Em A x HP1 houve diferenças somente para a variável REND $(\mathrm{P}<0,05)$.

Miranda Filho e Vencovsky (1984) relataram ser efeito de variedades $\left(\mathrm{v}_{\mathrm{i}}\right)$ o mais importante para a seleção de populações quando o efeito de heterose de variedades for não significativo. No presente estudo, seguindo o modelo dialélico parcial, o efeito de variedades foi decomposto em P1 e P2 e G.

A tabela 6 apresenta os efeitos genéticos de variedades $\left(\mathrm{v}_{\mathrm{i}}\right.$ e $\left.\mathrm{v}_{\mathrm{j}}\right)$, o efeito de heterose de variedades $\left(h_{i}\right.$ e $\left.h_{j}\right)$ e os valores de capacidade geral de combinação (CGC) para cada um dos sete parentais com relação as cinco variáveis analisadas

Com relação a CGC para a variável REND, observou-se no grupo de parentais 1 o maior valor para a população BR 106, assim como para as demais variáveis. Ainda para REND a população PC 9407 apresentou valor considerável $\left(36,7 \mathrm{~kg} \cdot \mathrm{ha}^{-1}\right)$. A população BR 106 apresenta como características predominantes o porte e ciclo de florescimento intermediários, sendo interessante o cruzamento com uma população que apresente CGCs baixas, ou até mesmo negativas para as variáveis FLOR e AP e, se possível, também AE. Para o grupo de parentais 2 uma atenção especial foi dada, não só para CGC, como também para os efeitos $v_{\mathrm{j}}$, uma vez que não foi detectada significância $(\mathrm{P}>0,05)$ através da análise dialélica conjunta, para a FV HP2 em quaisquer das cinco variáveis em estudo (Tabela 5). 
Tabela 6. Efeito de variedades $\left(v_{i}\right.$ e $\left.v_{j}\right)$, efeito de heterose de variedades $\left(h_{i}\right.$ e $\left.h_{j}\right)$ e estimativas de capacidade geral de combinação (CGC) das cinco variáveis analisadas em Londrina, Guarapuava, Ponta Grossa e Pato Branco. 2004/2005

\begin{tabular}{|c|c|c|c|c|c|c|c|c|c|c|c|c|c|c|c|}
\hline \multicolumn{16}{|l|}{ Grupo1 } \\
\hline & & & \multicolumn{2}{|c|}{ REND } & \multicolumn{3}{|c|}{ FLOR } & \multicolumn{3}{|c|}{$\mathbf{A P}$} & \multicolumn{3}{|c|}{$\mathbf{A E}$} & \multirow{2}{*}{\multicolumn{2}{|c|}{$\begin{array}{l}\text { PROL } \\
\text { (esp/pl) }\end{array}$}} \\
\hline & & & \multicolumn{2}{|c|}{$\left({ }^{1} \mathrm{~kg} \cdot \mathrm{ha}^{-1}\right)$} & \multicolumn{3}{|c|}{ (dias) } & \multicolumn{3}{|c|}{$(\mathrm{cm})$} & \multicolumn{2}{|c|}{$(\mathrm{cm})$} & & & \\
\hline & $\mathbf{v}_{\mathbf{i}}$ & $\mathbf{h}_{\mathbf{i}}$ & CGC & $\mathbf{v}_{\mathbf{i}}$ & $\mathbf{h}_{\mathbf{i}}$ & CGC & $\mathbf{v}_{\mathbf{i}}$ & $\mathbf{h}_{\mathbf{i}}$ & CGC & $\mathbf{v}_{\mathbf{i}}$ & $\mathbf{h}_{\mathbf{i}}$ & CGC & $\mathbf{v}_{\mathbf{i}}$ & $\mathbf{h}_{\mathbf{i}}$ & CGC \\
\hline PC 9407 & 360,8 & $-143,7$ & 36,70 & $-2,2$ & $-0,7$ & $-1,77$ & 4,9 & $-3,2$ & $-0,75$ & 0,3 & $-1,9$ & $-1,77$ & $-0,0350$ & $-0,0063$ & $-0,024$ \\
\hline PC 9501 & 388,3 & $-401,9$ & $-207,75$ & $-0,4$ & 0,1 & $-0,08$ & $-11,6$ & 2,0 & $-3,75$ & $-6,4$ & $-0,6$ & $-3,77$ & $-0,0025$ & $-0,0013$ & $-0,003$ \\
\hline BR 106 & $-749,0$ & 545,6 & 171,10 & 2,6 & 0,6 & 1,85 & 6,7 & 1,2 & 4,50 & 6,1 & 2,5 & 5,54 & 0,0375 & 0,0075 & 0,026 \\
\hline \multicolumn{16}{|l|}{ Grupo2 } \\
\hline & & & \multicolumn{2}{|c|}{$\left({ }^{1} \mathrm{~kg}^{\left.-h^{-1}\right)}\right.$} & \multicolumn{3}{|c|}{ (dias) } & & $(\mathrm{cm})$ & & \multicolumn{2}{|c|}{$(\mathrm{cm})$} & & \multicolumn{2}{|l|}{$\begin{array}{l}\text { PROL } \\
(\mathrm{esp} / \mathrm{pl})\end{array}$} \\
\hline & $\mathbf{v}_{\mathbf{j}}$ & $\mathbf{h}_{\mathbf{j}}$ & CGC & $\mathbf{v}_{\mathbf{j}}$ & $\mathbf{h}_{\mathbf{j}}$ & CGC & $\mathbf{v}_{\mathbf{j}}$ & $\mathbf{h}_{\mathbf{j}}$ & CGC & $\mathbf{v}_{\mathbf{j}}$ & $\mathbf{h}_{\mathbf{j}}$ & CGC & $\mathbf{v}_{\mathbf{j}}$ & $\mathbf{h}_{\mathbf{j}}$ & CGC \\
\hline PC 9701 & 356,6 & $-47,2$ & 131,10 & $-1,4$ & $-0,3$ & $-0,94$ & 2,3 & $-3,3$ & $-2,17$ & 0,1 & 1,2 & 1,25 & 0,0019 & $-0,0091$ & $-0,008$ \\
\hline PC 9502 & $-235,0$ & 174,3 & 56,80 & 0,4 & 0,3 & 0,48 & 3,3 & 2,4 & 4,08 & 8,6 & $-1,6$ & 2,67 & 0,0044 & 0,0014 & 0,004 \\
\hline PC 9702 & $-348,4$ & 38,7 & $-135,50$ & 1,1 & 0,1 & 0,65 & $-4,4$ & 2,8 & 0,58 & $-4,9$ & 3,6 & 1,17 & $-0,0081$ & 0,0134 & 0,009 \\
\hline PC 9703 & 226,8 & $-165,8$ & $-52,40$ & $-0,1$ & $-0,1$ & $-0,19$ & $-1,2$ & $-1,9$ & $-2,50$ & $-3,9$ & $-3,1$ & $-5,08$ & 0,0019 & $-0,0057$ & $-0,005$ \\
\hline
\end{tabular}

${ }^{1}$ corrigido para kg.ha ${ }^{-1}$ a $14,5 \%$ de umidade

Para REND os parentais PC 9701 e PC 9502 mostraram-se como promissores, por apresentar os maiores valores de CGC (131,1 e 56,8 $\mathrm{kg} \cdot \mathrm{ha}^{-1}$, respectivamente), assim como para a variável AE. Com relação a variável AP, estes apresentaram os maiores valores de $v_{j}$, enquanto que para FLOR foram baixos os valores de $v_{j}$ e CGC (Tabela 6). No entanto, quando se pretende trabalhar com cruzamentos em que se busca redução no ciclo de florescimento torna-se altamente viável o trabalho com parentais que apresentem valores baixos, ou até mesmo negativos para $\mathrm{v}_{\mathrm{j}}$ e CGC. Para PROL os valores calculados não foram considerados expressivos. Isto já era esperado, uma vez que os parentais utilizados não apresentavam mais de uma espiga por planta como característica preponderante. Dentro do contexto de CGC mereceram destaque as populações PC 9407 e BR 106 (Grupo 1), PC 9701 e PC 9502 (Grupo 2).

Analisando-se o efeito de capacidade específica de combinação (CEC) dos cruzamentos (Tabela 7), verificou-se que os híbridos BR 106 x PC 9701 e PC 9407 x PC 9502 apresentaram, além dos maiores valores de CEC para REND, as maiores médias de produtividade $(9.297$ e 9.018 kg.ha-1, respectivamente), como consta na Tabela 8 , em relação aos demais híbridos intervarietais testados nos quatro ambientes de estudo. Estes valores ficaram somente abaixo das testemunhas P 30F33 e IPR 115, sendo estes, híbridos simples de alta performance. Os híbridos BR 106 x PC 9701 e PC 9407 x PC 9502 apresentaram, também, porte relativamente baixo (223 cm para ambos), e ciclo de florescimento reduzido (72 e 70 dias, respectivamente). 
Tabela 7. Estimativas de capacidade específica de combinação (CEC) para os 12 híbridos avaliados em Londrina, Guarapuava, Ponta Grossa e Pato Branco. 2004/2005

\begin{tabular}{lccccc}
\hline Genótipo & REND & FLOR & AP & AE & PROL \\
\hline (Grupo 1 x Grupo 2) & $\left({ }^{1}{\mathrm{~kg} \cdot h \mathrm{ha}^{-1}}^{-}\right.$ & $($dias $)$ & $(\mathrm{cm})$ & $(\mathrm{cm})$ & $(\mathrm{esp} / \mathrm{pl})$ \\
\hline PC 9407 x PC 9701 & $-81,2$ & 1,4 & 1,7 & 0,4 & 0,019 \\
PC 9407 x PC 9502 & 135,3 & $-0,7$ & $-3,3$ & $-3,0$ & 0,002 \\
PC 9407 x PC 9702 & $-8,3$ & $-1,1$ & 1,9 & 1,5 & $-0,034$ \\
PC 9407 x PC 9703 & $-45,8$ & 0,4 & $-0,3$ & 1,0 & 0,013 \\
PC 9501 x PC 9701 & $-124,2$ & $-0,5$ & 0,4 & 3,4 & 0,003 \\
PC 9501 x PC 9502 & $-84,3$ & $-0,9$ & 4,9 & 4,5 & $-0,009$ \\
PC 9501 x PC 9702 & 96,9 & 0,9 & $-6,6$ & $-7,7$ & 0,010 \\
PC 9501 x PC 9703 & 111,7 & 0,5 & 1,3 & $-0,2$ & $-0,003$ \\
BR 106 x PC 9701 & 205,4 & $-0,9$ & $-2,1$ & $-3,9$ & $-0,021$ \\
BR 106 x PC 9502 & $-50,9$ & 1,6 & $-1,6$ & $-1,5$ & 0,007 \\
BR 106 x PC 9702 & $-88,5$ & 0,2 & 4,7 & 6,2 & 0,024 \\
BR 106 x PC 9703 & $-65,9$ & $-0,9$ & $-1,0$ & $-0,8$ & $-0,010$ \\
\hline
\end{tabular}

${ }^{1}$ corrigido para kg.ha-1 a $14,5 \%$ de umidade

Tabela 8. Médias dos tratamentos avaliados em Londrina, Guarapuava, Ponta Grossa e Pato Branco. Posicionamento com base no desempenho produtivo médio (REND). 2004/2005

\begin{tabular}{rlccccr}
\hline \multirow{2}{*}{ Pos Identificação } & & FLOR & AP & AE & PROL & REND \\
\cline { 3 - 6 } & & $($ dias $)$ & $(\mathrm{cm})$ & $(\mathrm{cm})$ & $(\mathrm{esp} / \mathrm{pl})$ & $\left.(\mathrm{kg.ha})^{-1}\right)$ \\
\hline 1 & P 30F33 & 71 & 209 & 121 & 0,99 & 10.452 \\
2 & IPR 115 & 71 & 223 & 127 & 1,01 & 9.975 \\
3 & BR 106 x PC 9701 & 72 & 223 & 131 & 1,00 & 9.297 \\
4 & PC 9407 x PC 9502 & 70 & 223 & 126 & 0,98 & 9.018 \\
5 & BR 106 x PC 9502 & 76 & 229 & 135 & 1,04 & 8.966 \\
6 & PC 9407 X PC 9701 & 71 & 221 & 128 & 0,99 & 8.876 \\
7 & BR 106 X PC 9703 & 73 & 223 & 128 & 1,01 & 8.842 \\
8 & BR 106 x PC 9702 & 75 & 232 & 141 & 1,06 & 8.736 \\
9 & PC 9407 x PC 9703 & 70 & 219 & 123 & 0,99 & 8.728 \\
10 & PC 9407 x PC 9702 & 70 & 224 & 129 & 0,96 & 8.682 \\
11 & BRS 1010 & 73 & 215 & 119 & 1,13 & 8.679 \\
12 & PC 9501 x PC 9703 & 72 & 218 & 119 & 0,99 & 8.641 \\
13 & PC 9501 x PC 9701 & 70 & 217 & 129 & 0,99 & 8.588 \\
14 & PC 9501 X PC 9502 & 71 & 228 & 132 & 0,99 & 8.554 \\
15 & PC 9501 x PC 9702 & 73 & 213 & 118 & 1,02 & 8.543 \\
16 & PC 9701 & 69 & 216 & 122 & 0,97 & 8.451 \\
17 & PC 9703 & 70 & 213 & 118 & 0,97 & 8.321 \\
18 & PC 9502 & 71 & 217 & 131 & 0,97 & 7.859 \\
19 & PC 9501 & 73 & 215 & 126 & 0,99 & 7.759 \\
20 & PC 9702 & 72 & 209 & 117 & 0,96 & 7.746 \\
21 & PC 9407 & 71 & 231 & 133 & 0,96 & 7.732 \\
22 & BR 106 & 75 & 233 & 139 & 1,03 & 6.622 \\
\hline
\end{tabular}


Com base nos gráficos 1 e 2, é possível visualizar que, além de outros fatores, para a formação de híbridos de alta produtividade de grãos, se faz necessário altos valores de CGC dentro de cada grupo de avaliação, ou seja, alta representatividade de efeitos aditivos, assim como altos valores de CEC dos cruzamentos, ou seja, efeitos não aditivos bem pronunciados.

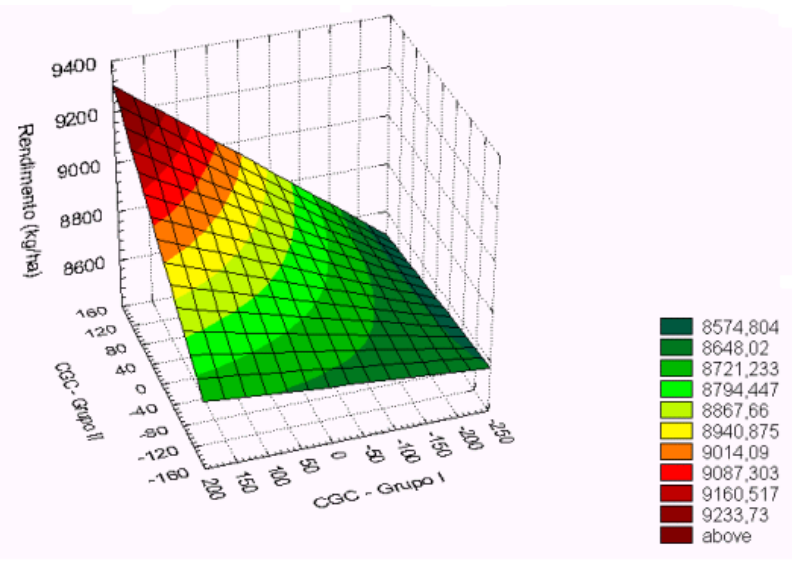

Gráfico 1. Plotagem quanto produtividade média (REND) dos híbridos nos 4 ambientes de estudo e estimativas de CGC para cada um dos grupos (CGC-Grupo 1e CGCGrupo2)

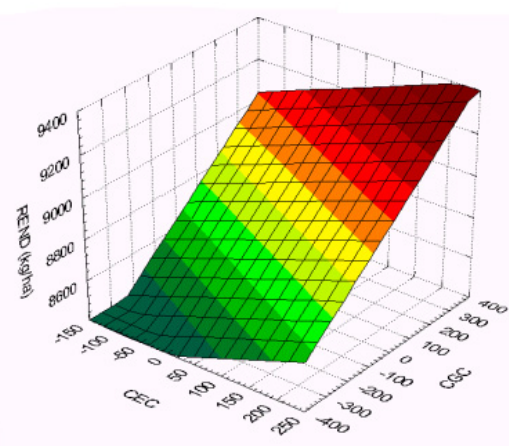
8581,82 8763,636 8854,545 8945,454 9036,363 9127,27
9218,18 9218,18 above

Gráfico 2. Plotagem quanto produtividade média (REND) dos híbridos nos 4 ambientes de estudo, somatória das estimativas de CGC para cada um dos grupos (CGC-Grupo 1e CGC-Grupo2) e estimativa da CEC.

\section{Conclusões}

Esses resultados permitem inferir que as populações PC 9701, BR 106, PC 9502 e PC 9407 são as mais promissoras fontes para a extração de linhagens, visando composição de híbridos de milho superiores.

Demonstrou-se o alto potencial dos parentais que, quando em cruzamento, originaram híbridos intervarietais com boas características agronômicas que podem, futuramente, serem usados em escala comercial.

É viável a utilização das populações PC 9701, BR 106, PC 9502 e PC 9407, para síntese de compostos com considerável produtividade e, também, para trabalhos com seleção recorrente, em especial, a recorrente recíproca.

\section{Referências}

ARAÚJO, P. M.; MIRANDA FILHO, J. B. Analysis of diallel cross for the evaluation of maize populations across enviroments. Crop Breeding And Applied Biotechnology, Londrina, v.1, n.3, p.255-262, 2001.

ARAÚJO, P. M.; PATERNIANI, E. Melhoramento de populações. In: DESTRO, D.; MONTALVÁN, R. Melhoramento Genético de Plantas. Londrina: EDUEL, 1999. p.311-330.

COMSTOCK, R. E.; ROBINSON, H. F.; HARVEY, P. H. A breeding procedure designed to make maximum use of both general and especific combining ability. Agronomy Journal, Madison, v.41, p.360-367, 1949.

COMSTOCK, R.E.; ROBINSON, H.F. The components of genetic variance in populations of biparental progenies and their use in estimating the average degree of dominance. Biometrics, Washington, v.4, p.254-266, 1948.

CORRÊA, A.; SANTOS, C.; KIST, B. B.; REETZ, E.; BELING, R. R. Anuário brasileiro de milho 2004. Santa Cruz do Sul, RS: Ed. Gazeta Santa Cruz, 2004.

CROSSA, J.; TABA, S.; WELHAUSEN, E. J. Heterotic patterns among Mexican reces of maize. Crop Science, Madison, v.30, n.6, p.1182-1190, 1990.

CRUZ, C. D.; CARNEIRO, P. C. S. Modelos biométricos aplicados ao melhoramento genético. Viçosa: Ed. UFV, 2003. v.2. 
CRUZ, C. D. Genes Aplicativo Computacional em Genética e Estatística. Viçosa: Ed. UFV, 1997.

CRUZ, C. D.; REGAZZI, A. J. Modelos biométricos aplicados ao melhoramento genético. Viçosa: Ed. UFV, 1994. v.1.

FERREIRA, D. F. Aplicativo MAPGEN 3.0. Lavras: Ed. UFL, 1993.

GAMA, E. E. G.; HALLAUER, A. R.; LOPES, M. A.; PARENTONI, S. N.; SANTOS, M. X.; GUIMARÃES, P. E. O. Combining ability among fifteen early cycle maize populations. Brazilian Journal. of Genetics, Ribeirão Preto, v.18, n.4, p.569-577, 1995.

GARDNER, C. O.; EBERHART, S. A. Analysis and interpretation of the variety cross diallel and related populations. Biometrics, Washington, v.22, p.439 - 452, 1966.

GARDNER,C. O.; HARVEY, P. H.; COMSTOCK, R. E. Dominance of genes controlling quantitative characters in maize. Biometrics, Washington, v.45, p.186-191, 1953.

GERAGE, A. C.; ARAÚJO, P. M. Heterose de Híbridos e Populações de Milho Avaliados Através de Cruzamento Dialélico. In: CONGRESSO NACIONAL DE MILHO E SORGO, 24., 2002, Florianópolis. Anais... Sete Lagoas: Associação Brasileira de Milho e Sorgo, 2002. 1 CD-ROM.

GORGULHO, E. P; MIRANDA FILHO, J. B. Estudo da capacidade combinatória de variedades de milho no esquema de cruzamento dialélico parcial. Bragantia, Campinas, v.60, n.1, p.1-8, 2001.

GRIFFING, B. A. Concept of general and especific combining ability in relation to diallel crossing systems. Australian Journal of Biology Science, Melbourne, v.9, p.463-93, 1956.

HALLAUER, A. R.; MIRANDA FILHO, J. B. Quantitative genetics in maize breeding. 2 ed. Ames: Iowa State University Press, 1988.
HAYMAN, B. I. The Teory and analisys of Diallel Crosses. Genetics, Austin, v.39, p.789-809, 1954.

MIRANDA FILHO, J. B.; GERALDI, I.O. An adapted model for the analysis of partial diallel crosses. Revista Brasileira de Genética, Ribeirão Preto, v.7, p.667-668, 1984.

MIRANDA FILHO, J. B.; VENCOVSKY, R. Analysis of diallel crosses among open-pollinated varieties of maize (Zea mays L.). Maydica, Bergamo, v.29, p.217-234, 1984.

MORAIS, A. R.; OLIVEIRA, A.C.; GAMA, E. E. G.; SOUZA JÚNIOR, C. L. A method for combined analysis of the diallel crosses repetead in several environments. Pesquisa Agropecuária Brasileira, Brasília, v.26, p.371-381, 1991.

OLIVEIRA, A. C.; MORAIS, A. R.; SOUZA JUNIOR, C.L.; GAMA, E. E. G. Análise de cruzamentos dialélicos parciais repetidos em vários ambientes. Revista Brasileira de Genética, Ribeirão Preto, v.3, p.517-533, 1987.

RUSSEL, W. A.; BLACKBURN, D. J.; LAMKEY, K. R. Evaluation of a modified reciprocal recurrent selection procedure for maize improvement. Maydica, Bergamo, v.37, p.61-67, 1992.

PARANÁ. Secretaria da Agricultura e Abastecimento do Paraná. Comparativo da área plantada, colhida e intenção de plantio, produção obtida e a obter, dos produtos selecionados no Paraná - safra 04/05 e 05/06, Curitiba, ago, 2005. Disponível em: <http://www.pr.gov.br/ seab> Acessado em: 24 set. 2005.

VENCOVSKY, R. Herança quantitativa. In: PATERNIANI, E.; VIÉGAS, G. P. Melhoramento e produção de milho. Campinas: Fundação Cargill, 1987. v.1, p.137-214. 
